\title{
Development of CMG for Three-Axis Free Air Floating Dynamics Simulator
}

\author{
By Takuya KanzawA ${ }^{1)}$, Tatsuya ENDO ${ }^{1)}$, Hiroshi KAWAI ${ }^{1)}$, Ken FujIWARA ${ }^{1)}$, \\ Yasuharu KAKEHASHI $^{2)}$, Takeshi FUKUYAMA ${ }^{2)}$ and Naoki SASAKI ${ }^{2)}$ \\ 1) Japan Aerospace Exploration Agency, Tsukuba, Japan \\ 2) Advanced Engineering Services, Tsukuba, Japan
}

(Received July 27th, 2009)

\begin{abstract}
This paper presents the development of large CMGs for on-ground attitude maneuver tests. The sizing of the CMGs, the overall design of the CMG system, and the results of development tests are shown. The developed CMGs are mounted on a large "dynamics simulator" test facility, which can provide an on-ground test environment of three-axis free rotational motion via air floating. The maneuver demonstration tests are performed using a cluster of four CMGs. The results show that the CMG cluster is capable of maneuvering the dynamics simulator with high agility, and the gimbals are steered with accuracy. Finally, two kinds of feedforward control laws for agile and smooth maneuvers are proposed.
\end{abstract}

Key Words: CMG, Dynamics Simulator, Maneuver, Agility, Precision

\section{Nomenclature}

$\left\{\overrightarrow{\mathbf{b}}_{\mathrm{B}}\right\}:$ : Spacecraft body-fixed reference frame

I : Moment of inertia of spacecraft

$\omega \quad$ : Angular velocity of spacecraft

$\mathbf{H}_{\mathrm{C}} \quad$ : Total angular momentum by CMGs

$\mathbf{T}_{\mathrm{C}} \quad$ : Attitude control torque by CMGs

A : Jacobian matrix

$\mathrm{h}_{\mathrm{R}} \quad$ : Rotor angular momentum

$\delta_{\mathrm{i}} \quad:$ Gimbal angle $(\mathrm{i}=1 \sim 4)$

$\mathrm{s}(): \sin ()$, same as $\mathrm{c}()$ for $\cos ()$

\section{Introduction}

Control Moment Gyros (CMGs) are often favorable for achieving agile maneuvering capabilities for spacecraft, because they are capable of producing larger control torque than Reaction Wheels (RWs). Recently, CMGs have been employed for some observing missions that require agility of the spacecraft; for example, WorldView-1 (launched in 2007) requires a rapid slew rate of $4.5 \mathrm{deg} / \mathrm{s}$, WorldView-2 (launched in 2009) requires $3.5 \mathrm{deg} / \mathrm{s}$, and Pleiades-HR (to be launched in 2010) requires $3.0 \mathrm{deg} / \mathrm{s}$.

However, difficulties may still be encountered due to the hardware and software complexities of CMGs. One of the difficulties is the singularity problem, in which no control torque is produced along a certain direction. The many CMG steering algorithms to avoid or pass the singularity have been extensively studied ${ }^{1-4)}$. In addition to the study of steering algorithms, a test facility to demonstrate the on-orbit attitude maneuver of CMGs mounted on spacecraft will be needed ${ }^{5)}$. The validation and verification that will be performed using the test facility will enable us to ensure the development of an attitude control system employing CMGs. To realize the on-ground maneuver tests, JAXA developed a large "dynamics simulator" test facility, which can provide a test environment of free rotational motion about three axes via air floating. Also, large single gimbal-type CMGs (SG-CMGs) were developed to produce a large control torque for the dynamics simulator.

The purpose of this paper is to show that the developed dynamics simulator and the CMGs perform well in the agilily and precision maneuver tests. First, in Section 2, this paper discusses the sizing of the CMGs, the design of the CMG system, and the results of CMG development tests. Second, in Section 3, the results of examinations to obtain the fundamental characteristics of the dynamics simulator, on which a cluster of four CMGs is mounted, are shown. Third, in Section 4, the results of maneuver demonstration tests with agility are shown and the maneuver performances by the CMGs are evaluated. Finally, in Section 5, the pre-test analysis and numerical simulations are shown, where two kinds of feedforward control laws for agile and smooth maneuver are proposed.

\section{Development of Large CMGs}

\subsection{Sizing of CMGs}

The CMGs for the dynamics simulator are sized for middleto large-class spacecraft that require agile maneuverability. The equations of motion for a spacecraft equipped with a cluster of four CMGs are given in the body-fixed reference

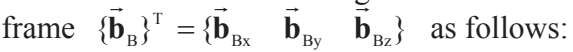

$$
\begin{gathered}
\mathbf{I} \dot{\boldsymbol{\omega}}+\widetilde{\boldsymbol{\omega}} \mathbf{I} \boldsymbol{\omega}=\mathbf{T}_{\mathrm{C}} \\
\dot{\mathbf{H}}_{\mathrm{C}}+\widetilde{\boldsymbol{\omega}} \mathbf{H}_{\mathrm{C}}=-\mathbf{T}_{\mathrm{C}}=\mathbf{T}_{\mathrm{CMG}}
\end{gathered}
$$

When the four CMGs mounted in a typical pyramid configuration are considered, as depicted in Fig. 1, the total angular momentum $\mathbf{H}_{\mathrm{C}}$ produced by the CMGs is expressed as: 


$$
\mathbf{H}_{\mathrm{C}}=\mathrm{h}_{\mathrm{R}}\left(\left[\begin{array}{c}
-\mathrm{c} \beta \mathrm{s} \delta_{1} \\
\mathrm{c} \delta_{1} \\
\mathrm{~s} \beta \mathrm{s} \delta_{1}
\end{array}\right]+\left[\begin{array}{c}
-\mathrm{c} \delta_{2} \\
-\mathrm{c} \beta \mathrm{s} \delta_{2} \\
\mathrm{~s} \beta \mathrm{s} \delta_{2}
\end{array}\right]+\left[\begin{array}{c}
\mathrm{c} \beta \mathrm{s} \delta_{3} \\
-\mathrm{c} \delta_{3} \\
\mathrm{~s} \beta \mathrm{s} \delta_{3}
\end{array}\right]+\left[\begin{array}{c}
\mathrm{c} \delta_{4} \\
\mathrm{c} \beta \mathrm{s} \delta_{4} \\
\mathrm{~s} \beta \mathrm{s} \delta_{4}
\end{array}\right]\right)
$$

It is assumed that the gimbals are steered simultaneously ( $\delta_{1}=\delta_{2}=\delta_{3}=\delta_{4} \equiv \delta$ ) and the spacecraft is controlled to perform the maneuver about the $\overrightarrow{\mathbf{b}}_{\mathrm{Bz}}$ axis. Then the maximum rotor angular momentum $\mathrm{h}_{\mathrm{R}}^{\max }$, which is required for one CMG, is simplified as follows:

$$
\mathrm{h}_{\mathrm{R}}^{\max }=\frac{\mathrm{I}_{\mathrm{z}} \omega_{\mathrm{z}}^{\max }}{4 \mathrm{~s} \beta \mathrm{s} \delta}
$$

The parameters for the spacecraft in Eq. (4) are selected as: moment of inertia $I_{z}=2000 \mathrm{kgm}^{2}$ and maximum slew rate $\omega_{\mathrm{z}}^{\max }=5.0 \mathrm{deg} / \mathrm{s}$. For the CMGs, the skew angle is selected as $\beta=54.7 \mathrm{deg}$ and the gimbal angle is assumed to be in the state of the $4 \mathrm{H}$ momentum saturation singularity ( $\delta=90 \mathrm{deg}$ ), in which the maximum total angular momentum is produced about the $\overrightarrow{\mathbf{b}}_{\mathrm{Bz}}$ axis. Therefore, the maximum angular momentum is calculated as $\mathrm{h}_{\mathrm{R}}^{\max } \approx 54 \mathrm{Nms}$.

The maximum gimbal angular velocity is determined to be $\dot{\delta}^{\max }=1.0 \mathrm{rad} / \mathrm{s}$. Therefore, the maximum gyroscopic output torque is determined to be $\mathrm{T}^{\max }=\mathrm{h}_{\mathrm{R}}^{\max } \cdot \dot{\delta}^{\max }=54 \mathrm{Nm}$.

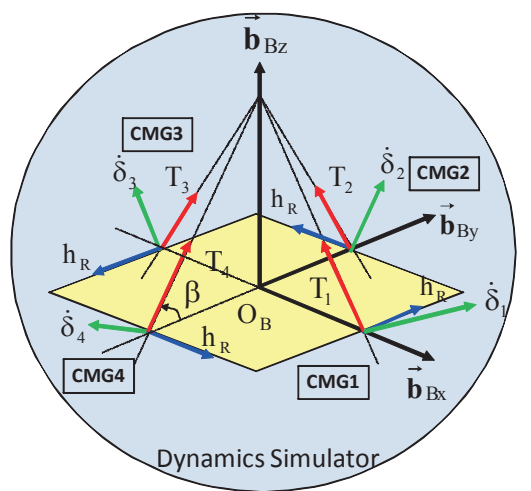

Fig. 1. Definition of the reference frame and configuration of CMGs.

\subsection{Design and development tests}

A set of five CMGs, a set of drivers and electronics, and a controller were designed and developed based on the established requirements. A CMG is shown in Fig. 2, and the simplified composition of the CMG system is shown in Fig. 3. Table 1 shows the main performance for the CMG.

Each CMG is composed of a rotor motor (brushless DC motor, 136210EC45, by MAXON), a high torque gimbal motor (AC servo motor, M-PS1012KN002, by NSK), an optical rotary encoder (contained in the rotor motor), a high-precision resolver (contained in the gimbal motor), a large fly-wheel, a casing, and so on. The fly-wheel is $262 \mathrm{~mm}$ in diameter, and its moment of inertia is approximately $0.086 \mathrm{kgm}^{2}$. The rotor motor is connected with the fly-wheel via ball bearings and a flexible coupling. The gimbal motor can rotate without a slip ring because the signal and the power for the rotor motor and the encoder are fed by cables passing through the inside of the gimbal motor.
The drivers and electronics part is composed of a central computer (IPC-BX701P4-AC517, by CONTEC), five sets of rotor motor drivers, and five sets of gimbal motor drivers, and so on. It is able to drive the CMGs by the control modes of rotor angular velocity, gimbal angle, and gimbal angular velocity. Based on a command from the CMG controller, the central computer processes and sends the command to the rotor motor drivers and gimbal motor drivers. It also receives the telemetry signal from the CMG via the rotor motor drivers and gimbal motor drivers, and transfers it to the $\mathrm{CMG}$ controller.

The CMG controller is a portable PC that includes software with functions of attitude control law/CMG steering law generation, data acquisition/recording, and so on. It connects with the central computer via one USB cable. The operator can send the command of gimbal angular velocity in two ways; one is a simple function profile, such as a sinusoidal or rectangular wave, which is directly created by means of a GUI, and the other is a command created based on the CMG steering law.

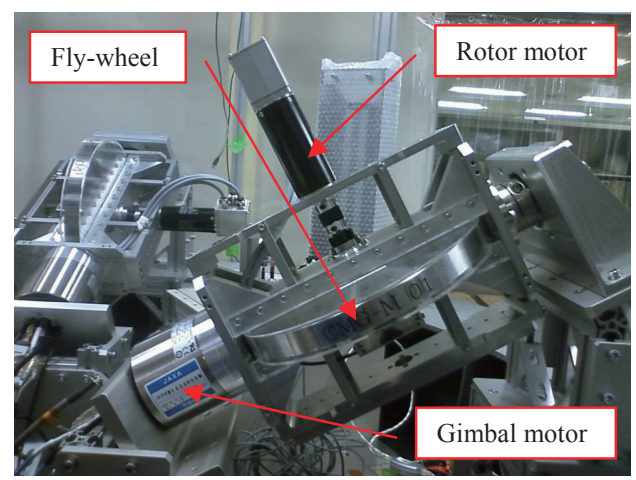

Fig. 2. View of a developed CMG.

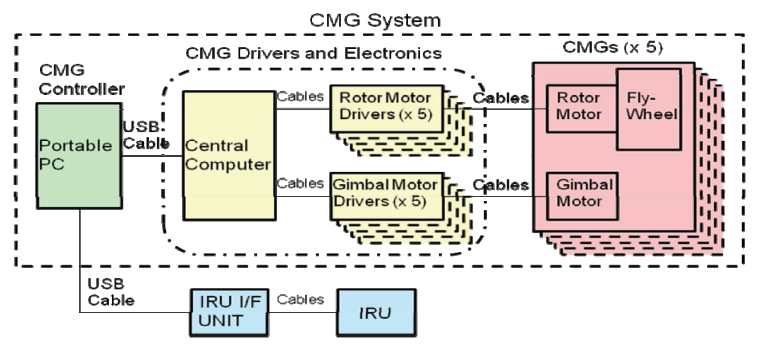

Fig. 3. Composition of the $\mathrm{CMG}$ system.

Table 1. Main Performance for CMG.

\begin{tabular}{|l|l|}
\hline Specification Item & Performance \\
\hline Max. rotor angular momentum & $54 \mathrm{Nms}(@ 6000 \mathrm{rpm})$ \\
\hline Max. rotor angular velocity & $6000 \mathrm{rpm}$ (limited by software) \\
\hline Resolution of sensor for rotor & More than 8 bits (optical encoder) \\
\hline Max. rotor motor torque & $0.625 \mathrm{Nm}$ \\
\hline Run-up time & Within 5 minutes up to $6000 \mathrm{rpm}$ \\
\hline Max. gimbal angular velocity & $1.0 \mathrm{rad} / \mathrm{s}$ (limited by software) \\
\hline Resolution of sensor for gimbal & More than 21 bits (resolver) \\
\hline Max. gimbal motor torque & $12 \mathrm{Nm}$ \\
\hline Max. gyroscopic output torque & $54 \mathrm{Nm}(54 \mathrm{Nms} \times 1 \mathrm{rad} / \mathrm{s})$ \\
\hline Power & $24 \mathrm{VDC}$ battery \\
\hline Mass & $27 \mathrm{~kg}$ \\
\hline
\end{tabular}


To ensure the development of high-torque and high-precision CMGs, several development tests are conducted on the manufactured CMGs as described below.

\section{Output torque measurement test}

The magnitude of the $\mathrm{CMG}$ gyroscopic output torque is directly measured to verify that it reaches the required value of $54 \mathrm{Nm}$. The CMG is placed on the Multi-component Force Plate (9253B12, by KISTLER), which can be used to measure the three-axis force and torque component. The rotor is rotated at the maximum angular velocity of $6000 \mathrm{rpm}$ ( $54 \mathrm{Nms})$, and the gimbal is continuously rotated by the rectangular wave of maximum angular velocity $\pm 1.0 \mathrm{rad} / \mathrm{s}$. Figure 4 shows that the output torque for both the $T_{x}$ and $T_{z}$ components in the force plate coordinate reaches the expected value of $54 \mathrm{Nm}$.

\section{Microvibration disturbance measurement test}

The microvibration disturbances generated by the $\mathrm{CMG}$ are transferred to the spacecraft body and induce a jitter, which degrades the spacecraft pointing performance. One of the significant microvibration disturbances is caused by the fly-wheel mass imbalance. After precision balancing of the fly-wheel, microvibrations due to the residual imbalance are measured to validate the design and the manufacturing of the rotor axis using the force plate. In the test, the magnitude of the force caused by static imbalance and the torque caused by dynamic imbalance are measured over the operating range of $6000 \mathrm{rpm}$ in cases of the gimbal servo being on or off. Figure 5 illustrates the waterfall chart. At the maximum value of $6000 \mathrm{rpm}$, the magnitude of the static imbalance force is about $10 \mathrm{~N}$, and the dynamic imbalance torque is about $0.5 \mathrm{Nm}$. The estimated static imbalance is about $4 \mathrm{gcm}$ less than the expected $10 \mathrm{gcm}$, and the dynamic imbalance is about 16 $\mathrm{gcm}^{2}$ less than the expected $100 \mathrm{gcm}^{2}$.

\section{Control accuracy measurement test}

Errors in total angular momentum and control torque produced by the CMG cluster affect the attitude control accuracy of the spacecraft. The errors come from the control accuracy of the rotor angular velocity achieved by the selected rotor motor/encoder, and the control accuracy of the gimbal angle and/or angular velocity achieved by the selected gimbal motor/resolver. The accuracy/stability for both the angular momentum and the output torque for each CMG are defined, and the results of evaluation are summarized in Table 2 . The output torque accuracy $\Delta \mathrm{T}_{\mathrm{G}}(3 \sigma)$ is less than 1 percent of the maximum output torque of $54 \mathrm{Nm}$.
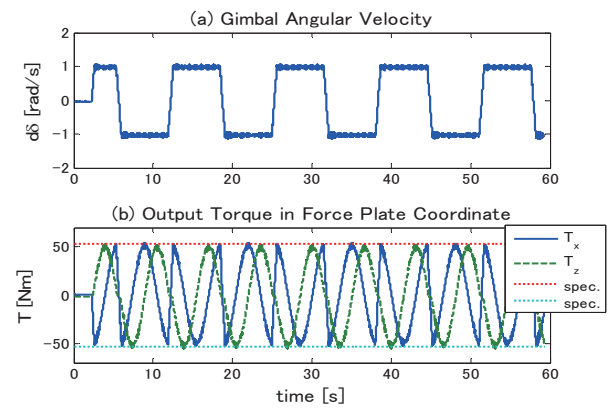

Fig. 4. Results of the output torque measurement test.

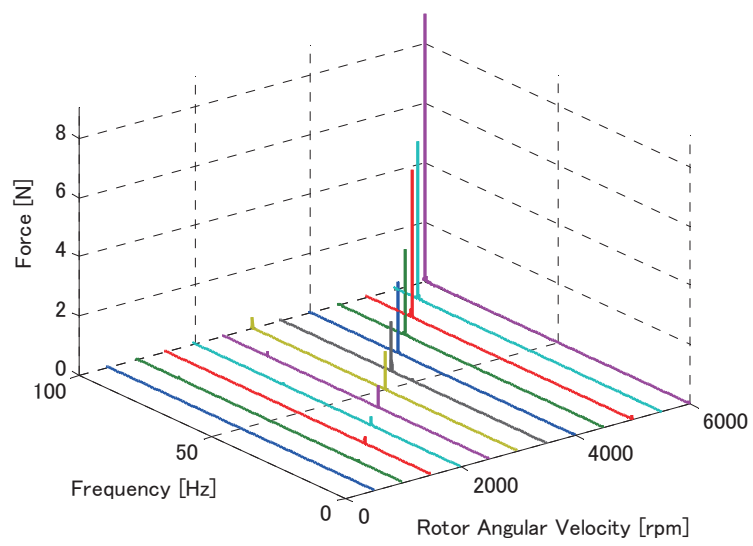

Fig. 5. Results of the microvibration disturbance measurement test.

Table 2. Evaluation of Accuracy/Stability for the CMG.

\begin{tabular}{|l|l|l|}
\hline Evaluation Item & Definition & Error Values \\
\hline $\begin{array}{l}\text { Rotor angular } \\
\text { velocity accuracy }\end{array}$ & $\begin{array}{c}\Delta \omega_{\mathrm{R}}[\mathrm{rpm}] \\
(@ 6000 \mathrm{rpm})\end{array}$ & $\begin{array}{l}5.49(\text { mean }) \\
3.22(3 \sigma)\end{array}$ \\
\hline $\begin{array}{l}\text { Angular momentum } \\
\text { accuracy }\end{array}$ & $\begin{array}{c}\Delta \mathrm{H}_{\mathrm{R}}=\mathrm{I}_{\mathrm{R}} \Delta \omega_{\mathrm{R}}[\mathrm{Nms}] \\
(@ 6000 \mathrm{rpm})\end{array}$ & $\begin{array}{l}0.09(\text { mean }) \\
0.03(3 \sigma)\end{array}$ \\
\hline $\begin{array}{l}\text { Output torque } \\
\text { accuracy }\end{array}$ & $\Delta \mathrm{T}_{\mathrm{R}}=\mathrm{I}_{\mathrm{R}} \Delta \omega_{\mathrm{R}} \dot{\delta}^{\max }[\mathrm{Nm}]$ & $\begin{array}{l}0.09(\text { mean }) \\
0.03(3 \sigma)\end{array}$ \\
\hline $\begin{array}{l}\text { Gimbal angle } \\
\text { accuracy }\end{array}$ & $\Delta \delta[\mathrm{deg}]$ & $\begin{array}{l}1.17 \times 10^{-4}(\mathrm{mean}) \\
4.87 \times 10^{-4}(3 \sigma)\end{array}$ \\
\hline $\begin{array}{l}\text { Output torque } \\
\text { stability }\end{array}$ & $\Delta \mathrm{T}_{\mathrm{S}}=\mathrm{h}_{\mathrm{R}}^{\max } \dot{\delta}_{\mathrm{m}}[\mathrm{Nm}]$ & $\begin{array}{l}0.04(\text { mean }) \\
0.31(3 \sigma)\end{array}$ \\
\hline $\begin{array}{l}\text { Angular momentum } \\
\text { stability }\end{array}$ & $\Delta \mathrm{H}_{\mathrm{S}}=\mathrm{h}_{\mathrm{R}}^{\max } \Delta \delta[\mathrm{Nms}]$ & $\begin{array}{l}1.12 \times 10^{-4}(\mathrm{mean}) \\
5.51 \times 10^{-4}(3 \sigma)\end{array}$ \\
\hline $\begin{array}{l}\text { Gimbal angular } \\
\text { velocity accuracy }\end{array}$ & $\Delta \dot{\delta}[\mathrm{rad} / \mathrm{s}]$ & $\begin{array}{l}0.01(\text { mean }) \\
(@ 1 \mathrm{rad} / \mathrm{s})\end{array}$ \\
\hline $\begin{array}{l}\text { Output torque } \\
\text { accuracy }\end{array}$ & $\Delta \mathrm{T}_{\mathrm{G}}=\mathrm{h}_{\mathrm{R}}^{\max } \Delta \dot{\delta}[\mathrm{Nm}]$ & $\begin{array}{l}0.47(\text { mean }) \\
(@ 1 \mathrm{rad} / \mathrm{s})\end{array}$ \\
\hline
\end{tabular}

\section{Fundamental Characteristics of Dynamics Simulator}

Figure 6 shows the three-axis free air floating dynamics simulator. The dynamics simulator is composed of a large aluminum sphere, approximately $2 \mathrm{~m}$ in diameter and approximately $1000 \mathrm{~kg}$ in weight, and a large saucer. The sphere floats in the saucer on compressed air from many small holes embedded in the saucer. Because equipment such as attitude control sensors and actuators can be mounted inside the sphere, unconstrained full $360 \mathrm{deg}$ rotational motion about the three axes can be realized. Furthermore, the surface of the sphere is ground with high precision, so that the friction between the sphere and the saucer is sufficiently low and torque-free motion is realized. The dynamics simulator is the only test facility to provide three-axis free rotational motion of a sphere that has large inertia and structural rigidity. On the other hand, most of the test facilities utilizing spherical air bearings are constrained to rotate about one or two axes, because the equipment is mounted on the main structure supported by air bearings ${ }^{5}$. 
The sphere can be divided into two sections: an upper hemisphere and a lower hemisphere. The large volume inside the sphere accommodates the large CMGs. They are mounted on the interface plate, which corresponds to the $\overrightarrow{\mathbf{b}}_{\mathrm{Bx}}-\overrightarrow{\mathbf{b}}_{\mathrm{By}}$ plane shown in Fig. 1, in the lower hemisphere. The CMG drivers and electronics are also mounted under the interface plate, which has high stiffness (more than $100 \mathrm{~Hz}$ ) to avoid resonance at the maximum operating angular velocity of 6000 rpm. Figure 7 shows the cluster of four CMGs mounted in the pyramid configuration with a skew angle of $60 \mathrm{deg}$ in the lower hemisphere. As shown in Fig. 3, an IRU is available for monitoring the angular velocity of the dynamics simulator in the range of $\pm 2 \mathrm{deg} / \mathrm{s}$.

Examinations to obtain the fundamental dynamic parameters are performed using the lower hemisphere of the dynamics simulator integrated with four CMGs. As a result, the moment of inertia about the $\overrightarrow{\mathbf{b}}_{\mathrm{Bz}}$ axis is estimated to be approximately $I_{z}=330 \mathrm{kgm}^{2}$, and the loss torque due to the air bearings is estimated to be between 7 and $12 \mathrm{mNm}$. The natural frequency of a stable pendulum-like swing motion about the $\overrightarrow{\mathbf{b}}_{\mathrm{Bx}}$ and $\overrightarrow{\mathbf{b}}_{\mathrm{By}}$ axes due to the offset between the center of mass and the center of rotation is about $0.5 \mathrm{~Hz}$.

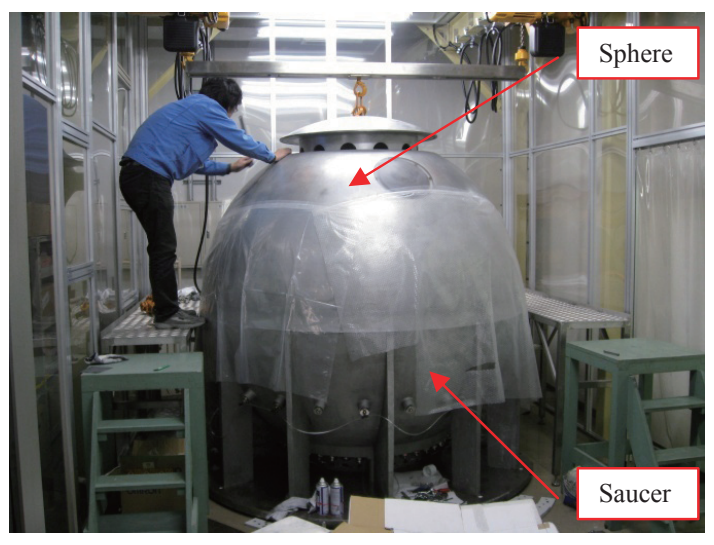

Fig. 6. View of the dynamics simulator.

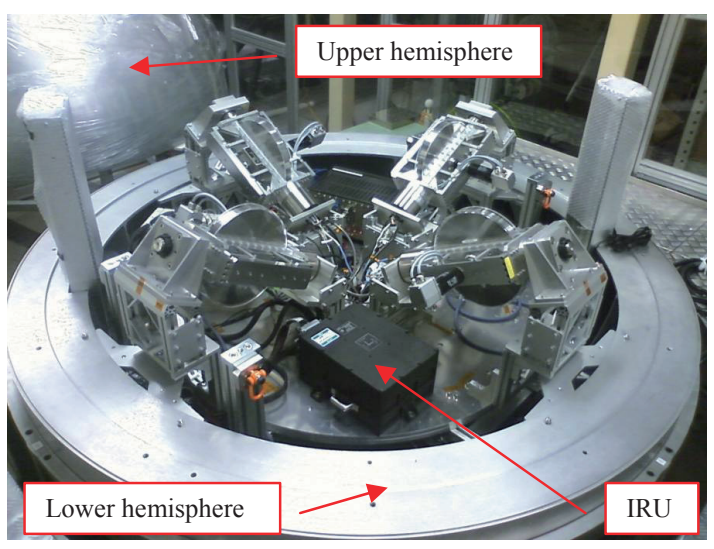

Fig. 7. Four CMGs mounted in the pyramid configuration.

\section{Maneuver Demonstration Test by CMG Cluster}

Using the lower hemisphere with the pyramid configuration of four CMGs, the attitude maneuvers about only the $\overrightarrow{\mathbf{b}}_{\mathrm{Bz}}$ axis are demonstrated. Figures 8 and 9 illustrate the results of the maneuvers in two ways. In the case of Fig. 8, the gimbals are simultaneously steered by the same continuous sinusoidal profile, which has a gimbal angular velocity of $0.1 \mathrm{rad} / \mathrm{s}$ in amplitude and $0.1 \mathrm{~Hz}$ in frequency. In the case of Fig. 9, the gimbals are also simultaneously steered by the same gimbal angular velocity calculated by the pseudo-inverse law $\left(\mathbf{A}^{+}=\mathbf{A}^{\mathrm{T}}\left(\mathbf{A A}^{\mathrm{T}}\right)^{-1}\right)$ based on the feedforward control torque $\mathbf{T}_{\mathrm{C}}$. Each rotor is rotated at an angular momentum of $\mathrm{h}_{\mathrm{R}}=10 \mathrm{Nms}$ in these cases. These results show that the agile maneuvers of the dynamics simulator are accomplished. Because of the small loss torque, its maximum angular velocity indicated in Fig. 8(e) corresponds to the calculated value as $\omega_{\mathrm{z}} \approx \mathrm{H}_{\mathrm{Cz}} / \mathrm{I}_{\mathrm{z}} \approx 10.9 \mathrm{Nms} / 330 \mathrm{kgm}^{2} \approx 1.9 \mathrm{deg} / \mathrm{s}$. Similarly, Fig. 9 implies that the maximum angular velocity is $\omega_{\mathrm{z}} \approx \mathrm{H}_{\mathrm{Cz}} / \mathrm{I}_{\mathrm{z}} \approx 23.7 \mathrm{Nms} / 330 \mathrm{kgm}^{2} \approx 4.1 \mathrm{deg} / \mathrm{s}$. In addition, the gimbals are steered with accuracy without delayed responses, and the four CMGs produce the expected total angular momentum and control torque during the maneuver.

Next, the errors of total angular momentum and control torque caused by the relative error of the gimbals are analytically explained to evaluate the exact steering by the CMG cluster. For example, the gimbals of CMG1 and CMG3 are simultaneously steered in the absence of a gimbal drive in CMG2 and CMG4 $\left(\delta_{2}=\delta_{4}=\dot{\delta}_{2}=\dot{\delta}_{4}=0\right)$. Then, the total angular momentum $\mathbf{H}_{\mathrm{C}}$ is described as follows based on Eq. (3):

$$
\mathbf{H}_{\mathrm{C}}=\mathbf{H}_{\mathrm{C}}^{\mathrm{ref}}+\Delta \mathbf{H}_{\mathrm{C}}^{\mathrm{G}}=\left[\begin{array}{c}
0 \\
0 \\
2 \mathrm{~h}_{\mathrm{R}} \mathrm{s} \beta \mathrm{s} \delta
\end{array}\right]+\mathrm{h}_{\mathrm{R}} \Delta \delta\left[\begin{array}{c}
\mathrm{c} \beta \mathrm{c} \delta \\
\mathrm{s} \delta \\
\mathrm{s} \beta \mathrm{c} \delta
\end{array}\right]
$$

where $\Delta \delta\left(\delta_{1}=\delta, \delta_{3}=\delta+\Delta \delta\right)$ is defined as the relative gimbal angle error of CMG3 with respect to CMG1. The first term is the ideal total angular momentum only about the $\overrightarrow{\mathbf{b}}_{\mathrm{Bz}}$ axis. The second term is the error of total angular momentum about the three axes due to error $\Delta \delta$. The error $(3 \sigma)$ due to the second term is estimated to be in the order of 0.01 percent for the total angular momentum in the test results.

Similarly, the total control torque $\mathbf{T}_{\mathrm{CMG}}$ produced by CMG1 and CMG3 is described as follows based on Eq. (2):

$$
\begin{gathered}
\mathbf{T}_{\mathrm{CMG}}=\dot{\mathbf{H}}_{\mathrm{C}}^{\mathrm{ref}}+\widetilde{\boldsymbol{\omega}} \mathbf{H}_{\mathrm{C}}^{\mathrm{ref}}+\Delta \dot{\mathbf{H}}_{\mathrm{C}}^{\mathrm{G}}+\widetilde{\boldsymbol{\omega}} \Delta \mathbf{H}_{\mathrm{C}}^{\mathrm{G}} \\
=\left[\begin{array}{c}
0 \\
0 \\
2 \mathrm{~h}_{\mathrm{R}} \mathrm{s} \beta \mathrm{c} \delta \cdot \dot{\delta}
\end{array}\right]+\left[\begin{array}{c}
2 \mathrm{~h}_{\mathrm{R}} \mathrm{s} \beta \mathrm{s} \delta \cdot \omega_{\mathrm{y}} \\
-2 \mathrm{~h}_{\mathrm{R}} \mathrm{s} \beta \mathrm{s} \delta \cdot \omega_{\mathrm{x}} \\
0
\end{array}\right] \\
+\mathrm{h}_{\mathrm{R}}\left[\begin{array}{c}
\mathrm{c} \beta(\mathrm{c} \delta \cdot \Delta \dot{\delta}-\mathrm{s} \delta \cdot \Delta \delta \cdot \dot{\delta}) \\
\mathrm{s} \delta \cdot \Delta \dot{\delta}+\mathrm{c} \delta \cdot \Delta \delta \cdot \dot{\delta} \\
\mathrm{s} \beta(\mathrm{c} \delta \cdot \Delta \dot{\delta}-\mathrm{s} \delta \cdot \Delta \delta \cdot \dot{\delta})
\end{array}\right]+\mathrm{h}_{\mathrm{R}} \Delta \delta\left[\begin{array}{c}
-\omega_{\mathrm{z}} \mathrm{s} \delta+\omega_{\mathrm{y}} \mathrm{s} \beta \mathrm{c} \delta \\
\omega_{\mathrm{z}} \mathrm{c} \beta \mathrm{c} \delta-\omega_{\mathrm{x}} \mathrm{s} \beta \mathrm{c} \delta \\
-\omega_{\mathrm{y}} \mathrm{c} \beta \mathrm{c} \delta+\omega_{\mathrm{x}} \mathrm{s} \delta
\end{array}\right]
\end{gathered}
$$

The first term is the ideal total torque only about the $\overrightarrow{\mathbf{b}}_{\mathrm{Bz}}$ axis. The second term is the gyroscopic torque caused by the interaction between the pendulum-like swing motion $\left(\omega_{x}, \omega_{y}\right)$ of the dynamics simulator and the total angular momentum $2 h_{R} s \beta s \delta$ of the two CMGs. Note that the magnitude of the gyroscopic torque is maximized at the saturation singularity 
( $\delta=90 \mathrm{deg})$. As seen in Fig. 8(e), the swing motion is suppressed within a small range during the maneuver. The third term is the torque error due to both the relative error of gimbal angle $\Delta \delta$, and the gimbal angular velocity $\Delta \dot{\delta}$. The error $(3 \sigma)$ due to the third term is estimated to be in the order of 1 percent for the total control torque in the test results. The fourth term is the torque error due to the gyroscopic interaction between the attitude motion $\left(\omega_{x}, \omega_{y}, \omega_{z}\right)$ and the angular momentum error of the second term in Eq. (5).

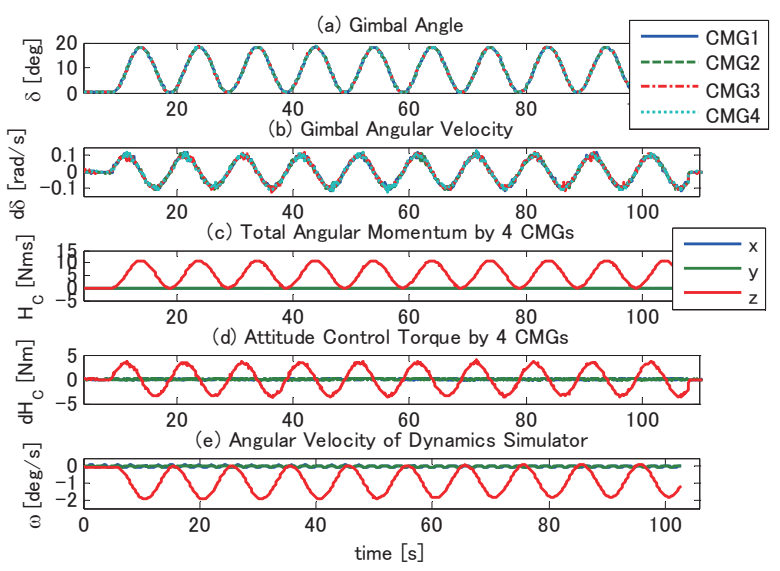

Fig. 8. Result of maneuver demonstration test (sinusoidal profile).

(a) Gimbal Angle
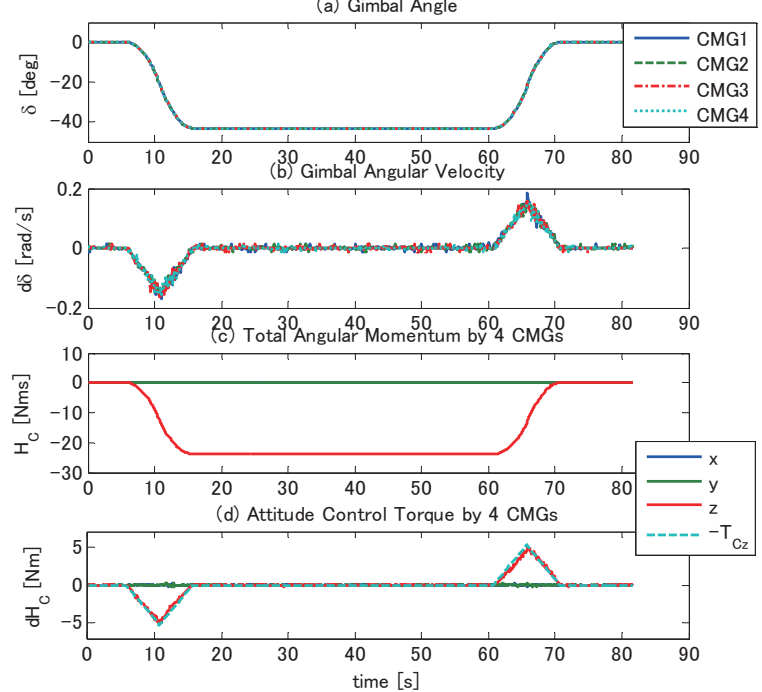

Fig. 9. Result of maneuver demonstration test (pseudo-inverse law).

\section{Feedforward Control Laws for Agile Maneuver}

In this section, two kinds of feedforward control laws are proposed and preliminary analysis is conducted prior to the maneuver tests using the four CMGs mounted on the dynamics simulator. These control laws are focused on the agility and smoothness of the maneuver.

\section{Case (i) The shortest path maneuver about the eigen-axis}

The spacecraft is controlled to maneuver along the shortest angular path about Euler's eigen-axis from the initial orientation to the target orientation. The desired maneuver profile is created using the eigen-angle $\Phi$ about the eigen-axis $\mathbf{e}$. In the profile, the time derivative of the commanded control torque (jerk) is taken into account for the boundary conditions in the initial orientation and the target orientation. This implies that the boundary conditions including jerk are effective to generate a smooth torque profile, and it will result in mitigating the vibration of the spacecraft due to the flexible appendages. If a seven-degree polynomial is chosen for the interpolation of the profile of the eigen-angle $\Phi$, then the four boundary conditions for $\Phi, \dot{\Phi}, \ddot{\Phi}$, and $\dddot{\Phi}$ in each orientation can be specified. Therefore, the commanded control torque $\mathbf{T}_{\mathrm{C}}$ acts about the eigen-axis $\mathbf{e}$ using profile $\Phi$ as follows:

$$
\begin{gathered}
\mathbf{T}_{\mathrm{C}}=\mathrm{I}_{\mathrm{e}} \ddot{\Phi} \cdot \mathbf{e} \\
=\mathrm{I}_{\mathrm{e}}\left(12 \mathrm{p}_{4} \mathrm{t}^{2}+20 \mathrm{p}_{5} \mathrm{t}^{3}+30 \mathrm{p}_{6} \mathrm{t}^{4}+42 \mathrm{p}_{7} \mathrm{t}^{5}\right) \cdot \mathbf{e}
\end{gathered}
$$

where $I_{e}$ is the moment of inertia about the eigen-axis, and $\mathrm{p}_{4}, \mathrm{p}_{5}, \mathrm{p}_{6}, \mathrm{p}_{7}$ are the coefficients depending on the total amount of eigen-angle $\Phi_{\mathrm{m}}$ and the maneuver finish time $\mathrm{t}_{\mathrm{m}}$.

Case (ii) Agile maneuver utilizing $\mathbf{4 H}$ singularity surface

The $4 \mathrm{H}$ momentum saturation singularity ${ }^{2)}$ is available for producing a rapid slew rate. Figure 10 shows the saturation singularity surface representing the envelope of the maximum available angular momentum in the pyramid configuration of the four CMGs with a skew angle of $60 \mathrm{deg}$. The duration of the maneuver is divided into three phases of acceleration, constant rate slewing, and deceleration. When the gimbals are held to produce the $4 \mathrm{H}$ maximum angular momentum during the slewing phase, the maximum slew rate capability $\omega_{\mathrm{Su}}$ of the spacecraft can be written as follows:

$$
\omega_{\mathrm{Su}}=\frac{\mathrm{H}_{\mathrm{Su}}}{\mathrm{I}_{\mathrm{u}}}=\frac{\mathrm{h}_{\mathrm{R}} \mathbf{u}^{\mathrm{T}} \mathbf{h}_{\mathrm{S}}}{\mathrm{I}_{\mathrm{u}}}
$$

where $\mathbf{u}$ is the direction of a given maneuver axis, $\mathbf{h}_{\mathrm{s}}$ is the analytical expression for the singularity surface ${ }^{6}, \mathrm{H}_{\mathrm{Su}}$ is the magnitude of the $4 \mathrm{H}$ angular momentum about the maneuver axis, and $I_{u}$ is the moment of inertia about the maneuver axis. The desired maneuver profile is created using the maneuver angle $\Theta$ about the maneuver axis $\mathbf{u}$. For the same reason as stated in Case (i), the maneuver angle is interpolated by a six-degree polynomial in the acceleration phase, a one-degree polynomial in the slewing phase, and again a six-degree polynomial in the deceleration phase. Therefore, the commanded control torque $\mathbf{T}_{\mathrm{C}}$ acts about the maneuver axis $\mathbf{u}$ using profile $\Theta$ as follows:

$$
\begin{aligned}
& \mathbf{T}_{\mathrm{C}}=\mathrm{I}_{\mathrm{u}} \ddot{\Theta} \cdot \mathbf{u} \\
& =\left\{\begin{array}{cc}
I_{u}\left(12 p_{14} t^{2}+20 p_{15} t^{3}+30 p_{16} t^{4}\right) \cdot u & \left(0 \leq t \leq t_{1}\right) \\
0 & \left(t_{1} \leq t \leq t_{2}\right) \\
I_{u}\left(12 p_{34} t_{d}{ }^{2}+20 p_{35} t_{d}{ }^{3}+30 p_{36} t_{d}{ }^{4}\right) \cdot u & \left(t_{2} \leq t \leq t_{m}\right)
\end{array}\right.
\end{aligned}
$$

where $t_{d}=t-t_{m}$, and $p_{14}, p_{15}, p_{16}, p_{34}, p_{35}, p_{36}$ are the coefficients depending on the constant slew rate $\omega_{\mathrm{Su}}$, slewing start time $t_{1}$, finish time $t_{2}$, and maneuver finish time $t_{m}$. CMG steering law

The Generalized Singularity Robust Inverse (GSRI) method is applied to calculate the commanded gimbal angular velocity $\dot{\boldsymbol{\delta}}$ as follows based on Eq. (2): 


$$
\dot{\boldsymbol{\delta}}=\mathbf{A}^{\mathrm{T}}\left(\mathbf{A A}^{\mathrm{T}}+\mathbf{V}\right)^{-1} \dot{\mathbf{H}}_{\mathrm{c}}
$$

The GSRI can be implemented online with a simple algorithm ${ }^{4}$. It allows rapid transition of singularities while the torque error occurs due to the increase of the weighting matrix $\mathbf{V}$ in the vicinity of singularities.

\section{Numerical simulations}

Next, numerical simulations are applied to the two kinds of feedforward control laws with the same steering law (GSRI), taking into account the multi-target observation mission of the spacecraft. Figure 11 shows the results of Case (i). The spacecraft maneuvers from the initial orientation $[0,0,0]$ deg to the first target orientation $[0,5,60]$ deg within $40 \mathrm{sec}$, and to the second target orientation $[0,0,-30]$ deg within $90 \mathrm{sec}$. The gimbals encounter the saturation singularity and the torque error occurs in the vicinity of $70 \mathrm{sec}$. Figure 12 shows the results of Case (ii). The spacecraft maneuvers about the first maneuver axis $\mathbf{u}_{1}=[0.044,-0.044,0.998]^{\mathrm{T}}$, and the second maneuver axis $\mathbf{u}_{2}=[-0.044,0.044,-0.998]^{\mathrm{T}}$. The four CMGs utilize the $4 \mathrm{H}$ angular momentum during the two constant slewing phases of $10-30 \mathrm{sec}$ and $60-80 \mathrm{sec}$. In these cases, the two successive maneuvers are achieved with agility and smoothness.

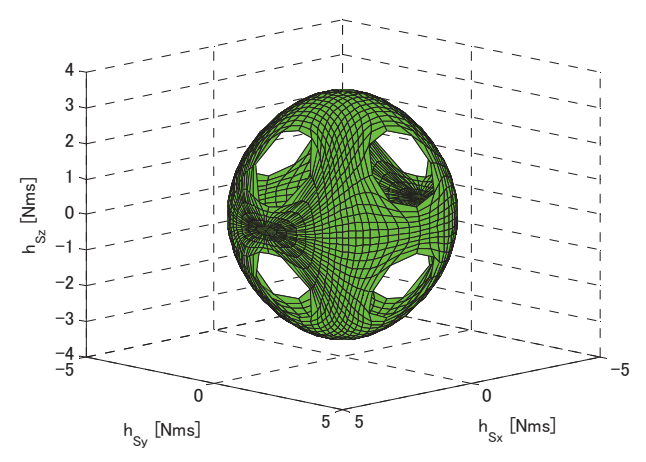

Fig. 10. Surface of $4 \mathrm{H}$ saturation singularity.
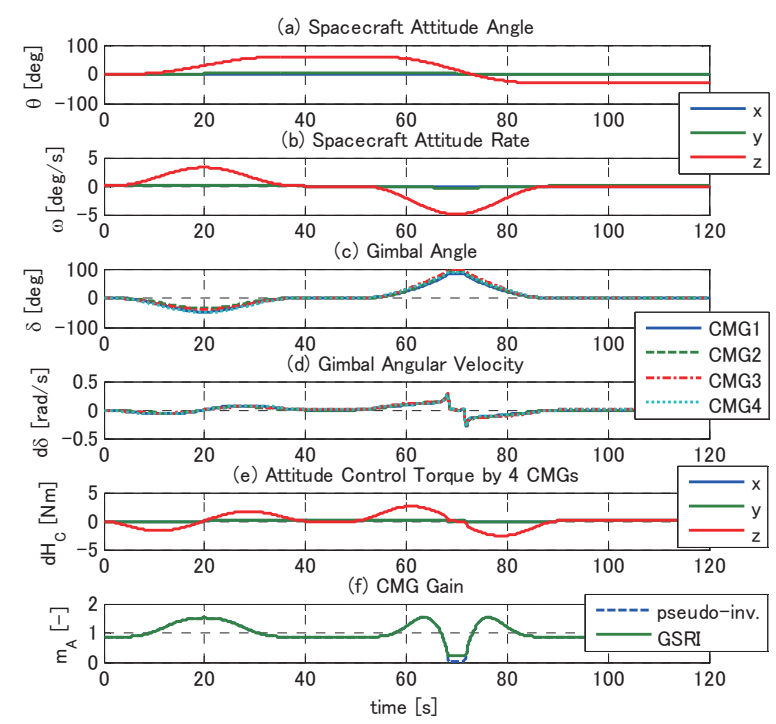

Fig. 11. The shortest path maneuver about the eigen-axis (Case (i)).
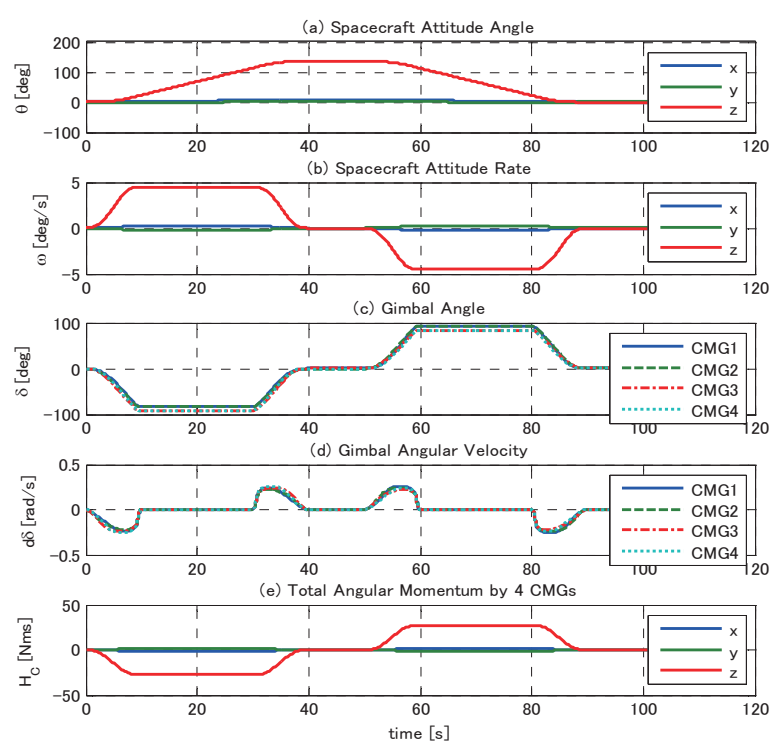

Fig. 12. Agile maneuver utilizing $4 \mathrm{H}$ saturation singularity (Case (ii)).

\section{Conclusion}

The results of CMG development tests showed that the $\mathrm{CMG}$ was able to produce large output torque of $54 \mathrm{Nm}$ with accuracy. The results of the maneuver demonstration test showed that the four CMGs were capable of maneuvering the dynamics simulator with high agility, and the gimbals were steered with accuracy. The loss torque due to air bearing and the swing motion due to offset were sufficiently low, and these did not influence the precision maneuver tests. During the maneuver, the four CMGs were able to produce the expected total angular momentum and control torque. Finally, through numerical simulations, it was shown that the proposed feedforward control laws were effective in accomplishing agile and smooth maneuvers. These laws will be applied to the maneuver tests of the dynamics simulator.

\section{References}

1) Kurokawa, H.: Survey of Theory and Steering Laws of Single-Gimbal Control Moment Gyros, J. Guidance, Control, and Dynamics, 30 (2007), pp.1331-1340.

2) Asghar, S., Palmer, P. L. and Roberts, M.: Exact Steering Law for Pyramid-Type Four Control Moment Gyro Systems, AIAA 2006-6652.

3) Wie, B., Bailey, D. and Heiberg, C.: Singularity Robust Steering Logic for Redundant Single-Gimbal Control Moment Gyros, J. Guidance, Control, and Dynamics, 24 (2001), pp.865-872.

4) Jung, D. and Tsiotras, P.: An Experimental Comparison of CMG Steering Control Laws, AIAA 2004-5294.

5) Schwartz, J. L., Peck, M. A. and Hall, C. D.: Historical Review of Air-Bearing Spacecraft Simulators, J. Guidance, Control, and Dynamics, 26 (2003), pp.513-522.

6) Wie, B.: Singularity Analysis and Visualization for Single-Gimbal Control Moment Gyro Systems, J. Guidance, Control, and Dynamics, 27 (2004), pp.271-282. 